\title{
Flat Space Cosmology as a Model of Penrose's Weyl Curvature Hypothesis and Gravitational Entropy
}

\author{
Eugene Terry Tatum \\ 760 Campbell Ln. Ste. 106 \#161, Bowling Green, KY, USA \\ Email:ett@twc.com
}

How to cite this paper: Tatum, E.T. (2018) Flat Space Cosmology as a Model of Penrose's Weyl Curvature Hypothesis and Gravitational Entropy. Journal of Modern Physics, 9, 1935-1940. https://doi.org/10.4236/jmp.2018.910121

Received: July 2, 2018

Accepted: September 1, 2018

Published: September 4, 2018

Copyright $\odot 2018$ by author and Scientific Research Publishing Inc. This work is licensed under the Creative Commons Attribution International License (CC BY 4.0).

http://creativecommons.org/licenses/by/4.0/

\section{(c) (i) Open Access}

\begin{abstract}
FSC is shown to be an excellent model of Penrose's Weyl curvature hypothesis and his concept of gravitational entropy. The assumptions of FSC allow for the minimum entropy at the inception of the cosmic expansion and rigorously define a cosmological arrow of time. This is in sharp contrast to inflationary models, which appear to violate the second law of thermodynamics within the early universe. Furthermore, by virtue of the same physical assumptions applying at any cosmic time $t$, the perpetually-flat FSC model predicts the degree of scale invariance observed in the $\mathrm{CMB}$ anisotropy pattern, without requiring an explosive and exceedingly brief inflationary epoch. Penrose's concepts, as described in this paper, provide support for the idea that FSC models gravitational entropy and Verlinde's emergent gravity theory.
\end{abstract}

\section{Keywords}

Flat Space Cosmology, Cosmology Theory, Gravitational Entropy, Weyl's Curvature Hypothesis, Black Holes, Cosmic Inflation, Cosmic Flatness, Cosmic Microwave Background

\section{Introduction and Background}

If the expanding universe follows the second law of thermodynamics, then the total cosmic entropy of each earlier epoch in time must have had a lower value. The various theories of cosmic inflation appear to ignore this stipulation, as detailed by Roger Penrose in his 2016 book entitled, Fashion, Faith and Fantasy in the New Physics of the Universe [1]. Penrose makes a convincing argument that the gravitational entropy state of the earliest universe (i.e., before the supposed inflationary epoch at $10^{-37}$ to $10^{-32}$ second of cosmic time) must have been ex- 
ceedingly low, even in comparison to the post-inflationary universe. This is in stark contrast to the belief on the part of inflationists that inflation "solves" the problem posed by an extremely chaotic (i.e., high entropy) Big Bang quantum fluctuation event as a beginning of the universe. Yet, as Penrose points out, any such inflationary solution would be either a violation of the second law or an unnecessary solution to a non-existent problem (i.e., that inflation began in a smooth flat patch of primordial space-time). So, in Penrose's view, a theory of cosmic inflation is either a violation of physics or, at best, completely unnecessary. Others have expressed similar concerns with inflationary theory, including one of its founders [2].

In 1979, before any theories of inflation were proposed, Penrose first addressed the tension between the remarkable apparent homogeneity and isotropy of the universe (also inherent in the FLRW model) and the second law requirement of extremely low beginning entropy. He introduced the concept of "gravitational entropy", wherein the ongoing clustering of stars and galaxies, and the formation of black holes, is in the direction of ever-greater total cosmic entropy. Thus, in stark contrast to the thermal entropy of a gas, the total entropy of a gravitating system can be considered to be lowest at its smallest scale and its most homogeneous gravitational state. In his "Weyl curvature hypothesis" [3], Penrose associates the lowest entropy state of the earliest universe with a vanishing Weyl curvature tensor. Thus, if one wishes to consider the theoretical possibility of a "Big Bang" from a singularity condition, under this hypothesis, the universe begins "free of independent gravitational degrees of freedom" [Penrose (2016), pages 371-374]. Operationally, one can consider this to be a "zero" gravitational entropy state with respect to any future positive gravitational entropy values of the expanding universe. This gravitational entropy concept is a key feature of the Flat Space Cosmology (FSC) model [4] and Erik Verlinde's "emergent gravity" (entropic gravity) theory [5] [6]. The concept of gravitational entropy also allows for a very tight FSC correlation with the observed anisotropy magnitude $(0.66 \times$ $10^{-5} \mathrm{dT} / \mathrm{T}$ ) in the cosmic microwave background (CMB) [7].

The Weyl curvature hypothesis and the FSC model both provide for a cosmological arrow of time [8]. This is in contrast to the standard inflationary model, which has no effective means by which the law of entropy can be obeyed if the model starts with a highly chaotic and high entropy quantum fluctuation event.

One of the current sources of tension between the most recent CMB observations [9] and standard inflationary cosmology is the assertion of global cosmological spatial flatness and dark energy dominance. The assertion of flatness stipulates a Friedmann curvature $k$ term value of zero, while the assertion of dark energy dominance stipulates a small negative value to the $k$ term. Both stipulations cannot be true at the same time! Furthermore, while the near scale-invariance of the $\mathrm{CMB}$ power spectrum is commonly touted to be a validation of inflationary cosmology, scale invariance is even easier to explain in FSC. Scale invariance in a cosmological model essentially means that the same laws of physics apply to any 
scale of the cosmological model. Thus, one would expect a large degree of self-similarity between adjacent cosmological epochs in a spatially-flat model in which the same set of basic assumptions is prescribed to occur at any cosmological time $t$. The following section presents the current five basic assumptions of FSC.

\section{The Five Assumptions of FSC}

FSC models the Hawking-Penrose conjecture that a smoothly-expanding cosmic system beginning from a singularity can be modeled within the rules of general relativity as a time-reversed black hole. Hence, the assumptions of FSC are as follows:

1) The cosmic model is an ever-expanding sphere such that the cosmic horizon always translates at speed of light $c$ with respect to its geometric center at all times $t$. The observer is operationally-defined to be at this geometric center at all times $t$.

2) The cosmic radius $R_{t}$ and total mass $M_{t}$ follow the Schwarzschild formula $R_{t} \cong 2 G M_{t} / c^{2}$ at all times $t$.

3) The cosmic Hubble parameter is defined by $H_{t} \cong c / R_{t}$ at all times $t$.

4) Incorporating our cosmological scaling adaptation of Hawking's black hole temperature formula, at any radius $R_{t}$ cosmic temperature $T_{t}$ is inversely proportional to the geometric mean of cosmic total mass $M_{t}$ and the Planck mass $M_{p l} . R_{p l}$ is defined as twice the Planck length (i.e., as the Schwarzschild radius of the Planck mass black hole). With subscript $t$ for any time stage of cosmic evolution and subscript $p l$ for the Planck scale epoch, and, incorporating the Schwarzschild relationship between $M_{t}$ and $R_{t}$,

$$
\begin{aligned}
& k_{B} T_{t} \cong \frac{\hbar c^{3}}{8 \pi G \sqrt{M_{t} M_{p l}}} \cong \frac{\hbar c}{4 \pi \sqrt{R_{t} R_{p l}}} \\
& \left\{\begin{array}{ll}
M_{t} \cong\left(\frac{\hbar c^{3}}{8 \pi G k_{B} T_{t}}\right)^{2} \frac{1}{M_{p l}} & \text { (A) } \\
R_{t} \cong \frac{1}{R_{p l}}\left(\frac{\hbar c}{4 \pi k_{B}}\right)^{2}\left(\frac{1}{T_{t}}\right)^{2} & \text { (B) } \\
R_{t} T_{t}^{2} \cong \frac{1}{R_{p l}}\left(\frac{\hbar c}{4 \pi k_{B}}\right)^{2} & \text { (C) } \\
t \cong \frac{R_{t}}{c} & \text { (D) }
\end{array}\right\}
\end{aligned}
$$

5) Total entropy $S_{t}$ of the cosmic model follows the Bekenstein-Hawking black hole formula [10] [11], wherein $R_{t}$ is the cosmic radius at time $t$ and $L_{p}$ is the Planck length.

$$
S_{t} \cong \frac{\pi R_{t}^{2}}{L_{p}^{2}}
$$

These model assumptions correlate very closely with current observations, as 
detailed in "Clues to the Fundamental Nature of Gravity, Dark Energy and Dark Matter" and "Temperature Scaling in Flat Space Cosmology in Comparison to Standard Cosmology" [Tatum, et al. (2018)]. It should be remembered that all five of these assumptions apply to every second of the FSC cosmological model. Hence, scale invariance to the degree seen in the CMB anisotropy pattern is a prediction of FSC and must not be considered the exclusive domain of inflationary models.

\section{Perpetual Friedmann's Critical Density in FSC}

As described in some detail in the seminal FSC papers [12] [13] [14] [15], the first three assumptions allow for perpetual Friedmann's critical density (i.e., perpetual global spatial flatness) of the expanding FSC cosmological model from inception. By dividing the Schwarzschild mass (defined in terms of cosmic radius $R_{o}$ ) by the spherical volume, and substituting $c^{2} / R_{o}^{2}$ with $H_{o}^{2}$, Friedmann's critical mass density $\rho_{0}=\frac{3 H_{0}^{2}}{8 \pi G}$ is achieved for any given moment of observation (hence the subscript " $o$ ") in cosmic time. So, perpetual Friedmann's critical density (i.e., perpetual spatial flatness) from inception is a fundamental feature of the FSC model. For example, the current observational global Hubble parameter $H_{o}$ value is calculated by the FSC model to be $66.89325791854758 \mathrm{~km} \cdot \mathrm{s}^{-1} \cdot \mathrm{Mpc}^{-1}$, which fits the lower end range of the 2015 Planck Collaboration consensus observational value of $67.8+/-0.9 \mathrm{~km} \cdot \mathrm{s}^{-1} \cdot \mathrm{Mpc}^{-1}$ (68\% confidence interval), as shown in the FSC "Clues" paper in the July 2018 issue of Journal of Modern Physics.

\section{Gravitational Entropy in FSC}

FSC models the Hawking-Penrose conjecture that a smoothly-expanding cosmic system beginning from a singularity can be modeled within the rules of general relativity as a time-reversed black hole. Thus, assumption \#5 defining FSC entropy by $S_{t} \cong \frac{\pi R_{t}^{2}}{L_{p}^{2}}$ at all times $t$ seems appropriate. As of yet, there appear to be no known scale limitations of a cosmological model which follows the above assumptions. And yet, each successively earlier stage of the FSC model must have a lower (gravitational) entropy value by virtue of the assumption that the entropy of a black hole scales according to $R^{2}$. Thus, it is shown by the above theoretical considerations and model assumptions that FSC is a model of Penrose's Weyl curvature hypothesis, his concept of gravitational entropy, and Verlinde's theory of emergent gravity.

\section{Summary and Conclusions}

FSC has been shown to be an excellent model of Penrose's Weyl curvature hypothesis and his concept of gravitational entropy. The assumptions of FSC allow for the minimum entropy at the inception of the cosmic expansion and rigo- 
rously define a cosmological arrow of time. This is in sharp contrast to inflationary models, which appear to violate the second law of thermodynamics within the early universe. Furthermore, by virtue of the same physical assumptions applying at any cosmic time $t$, the perpetually-flat FSC model predicts the degree of scale invariance observed in the $\mathrm{CMB}$ anisotropy pattern, without requiring an explosive and exceedingly brief inflationary epoch. Penrose's concepts, as described in this paper, provide support for the idea that FSC models gravitational entropy and Verlinde's emergent gravity theory.

\section{Dedications and Acknowledgements}

This paper is dedicated to Dr. Stephen Hawking and Dr. Roger Penrose for their groundbreaking work on black holes and their possible application to cosmology. Dr. Tatum also thanks Dr. Rudolph Schild of the Harvard Center for Astrophysics for his past support and encouragement.

\section{Conflicts of Interest}

The authors declare no conflicts of interest regarding the publication of this paper.

\section{References}

[1] Penrose, R. (2016) Fashion Faith and Fantasy in the New Physics of the Universe. Princeton University Press, Princeton, US. https://doi.org/10.1515/9781400880287

[2] Steinhardt, P.J. (2011) Scientific American, 304, 18-25. https://doi.org/10.1038/scientificamerican0411-36

[3] Penrose, R. (1979) Singularities and Time-Asymmetry. In: Hawking, S.W. and Israel, W., Eds., General Relativity: An Einstein Centenary Survey, Cambridge University Press, Cambridge, 581-638.

[4] Tatum, E.T. and Seshavatharam, U.V.S. (2018) Journal of Modern Physics, 9, 1469-1483. https://doi.org/10.4236/jmp.2018.98091

[5] Verlinde, E. (2010) On the Origin of Gravity and the Laws of Newton. arXiv:1001.0785v1 [hep-th].

[6] Verlinde, E. (2016) Emergent Gravity and the Dark Universe. aeXiv:1611.02269v2 [hep-th].

[7] Tatum, E.T. (2018) Journal of Modern Physics, 9, 1484-1490. https://doi.org/10.4236/jmp.2018.98092

[8] Tatum, E.T. and Seshavatharam, U.V.S. (2018) Journal of Modern Physics, 9, 1404-1414. https://doi.org/10.4236/jmp.2018.97085

[9] Planck Collaboration XIII. (2016) Astronomy \& Astrophysics, 594, A13.

[10] Bekenstein, J.D. (1974) Physical Review D, 9, 3292-3300. https://doi.org/10.1103/PhysRevD.9.3292

[11] Hawking, S. (1976) Physical Review D, 13, 191-197. https://doi.org/10.1103/PhysRevD.13.191

[12] Tatum, E.T., Seshavatharam, U.V.S. and Lakshminarayana, S. (2015) International Journal of Astronomy and Astrophysics, 5, 116-124. https://doi.org/10.4236/ijaa.2015.52015 
[13] Tatum, E.T., Seshavatharam, U.V.S. and Lakshminarayana, S. (2015) Journal of Applied Physical Science International, 4, 18-26.

[14] Tatum, E.T., Seshavatharam, U.V.S. and Lakshminarayana, S. (2015) Frontiers of Astronomy, Astrophysics and Cosmology, 1, 98-104.

[15] Tatum, E.T., Seshavatharam, U.V.S. and Lakshminarayana, S. (2015) International Journal of Astronomy and Astrophysics, 5, 133-140.

https://doi.org/10.4236/ijaa.2015.53017 